\title{
Solution of the Hartree-Fock Equations ${ }^{\star}$
}

\author{
G. Fonte, R. Mignani and G. Schiffrer \\ Istituto di Fisica Teorica dell'Università, Catania (Italy) \\ Received December 4, 1972; in revised form May 16, 1973
}

\begin{abstract}
We suggest to use the Newton iteration method for constructing a (locally unique) solution of the atomic and nuclear Hartree-Fock equations for an arbitrary number of particles. Our proposal is based on a theorem by Kantorovič and rests on the following points: 1) the two-body potential must satisfy a boundedness condition; 2) the zero-order approximation, used to start the iteration sequence, must satisfy certain conditions, to be proved numerically. Condition 1) holds, for instance, for all local potentials, defined by a bounded function and for a class of nonlocal potentials; it does not hold for local potentials, behaving as $1 / r$ near the origin.
\end{abstract}

\section{Introduction}

Although the Hartree-Fock theory is known since the earliest years of quantum mechanics [1] and has been extensively used in atomic [2] and in nuclear physics [3], no rigorous and general treatment of the related equations has appeared so far in the literature. This gap has been filled only partially by some more recent papers [4-6]. Refs. [4] and $[5]^{1}$ are mathematically rigorous, but deal only with systems of two identical fermions plus another particle, the former reference with harmonic oscillator interactions, the latter with Coulomb ones (helium atom). In Ref. [6], the problem of computing a solution, proving its local uniqueness and estimating the error due to the finiteness of the iteration, has been solved for the nucleus ${ }^{16} \mathrm{O}$ in a subspace spanned by a finite harmonic oscillator basis, using the Newton method of successive approximations in the generalized form and with the convergence conditions of Kantorovič $[7,8]^{2}$. The aim of the present paper is to contribute to a better mathematical understanding of the problem, by extending the theory of Ref. [6] to an infinite-dimensional space and presenting it independently of any

* This work has been supported in part by Istituto Nazionale di Fisica Nucleare (Sezione di Catania) and by Centro Siciliano di Fisica Nucleare e di Struttura della Materia (Catania).

${ }^{1}$ We thank the referee for bringing to our attention this paper.

${ }^{2}$ Russian references are translitterated according to the international convention, recommended in Suppl. Nuovo Cim. 1, 387 (1955). 
particular representation; however, we shall not be concerned here with numerical calculations.

We write down the Hartree-Fock equations in sect. 2 as a single equation of the form $F(X)=0$, where $X$ is a vector of a suitable Hilbert space $\mathscr{H}$ and $F$ is a nonlinear operator, mapping $\mathscr{H}$ into itself. The vector $X$ contains all the unknown single-particle states and energies. The Newton-Kantorovic method can be employed when $F$ is twice Fréchet-differentiable [8] in a neighbourhood of a solution. In Section 3 we discuss three conditions on the two-body interaction, which are related to the existence and properties of the first and second Fréchet derivatives $F^{\prime}$ and $F^{\prime \prime}$ of $F$. Two examples of classes of potentials satisfying such conditions are given. In Section 4 we prove the existence of $F^{\prime}$ and $F^{\prime \prime}$. In Section 5 we discuss the application of Kantorovic theorem. We emphasize that the proof of existence and uniqueness of a solution based on this theorem can be achieved only by means of some numerical calculations: one has to choose an approximation $X_{0}$ to the expected solution and to check numerically that it satisfies the conditions of the theorem; in the successful case, we can be sure that a locally unique solution of the equations exists and it can be explicitly computed to any degree of accuracy. For easier reference of the reader, we report an abstract of the notation in the appendix.

We think that the proposed method is not only of an obvious relevance for mathematical physicists, but also for atomic and nuclear physicists interested in computational work: its main advantages are the very fast convergence, the possibility of estimating the error due to truncation of the basis and of the iteration; of using any representation; of solving more complicated problems, including constraints, correlations and so on $[6,9]$. Ref. [9] contains a review of the present and related work, including applications to nuclear physics. In Ref. [10] the problem of consistent symmetries has been treated in the framework of the Newton-Kantorovič method, which has provided in this case a significant clarification of the question about convergence conditions in presence of symmetries.

\section{Hartree-Fock Equations}

Considering a system of $A$ identical fermions, we denote by $h$ the Hilbert space of states of a single particle, by $h_{A}$ the $\operatorname{direct} \operatorname{sum} h \oplus h \oplus \ldots$ ( $A$ times), by $\mathscr{R}$ the real numbers, by $\mathscr{R}_{A}$ the direct sum $\mathscr{R} \oplus \mathscr{R} \oplus \ldots$ ( $A$ times). In order to simplify the writing, we shall consider only real Hilbert spaces; the extension to complex ones is straightforward, by taking the real and the imaginary part of the equations. The HartreeFock equations will be written down as a single equation in the Hilbert 
space $\mathscr{H}=h_{A} \oplus \mathscr{R}_{A}$ :

$$
[(T-\Lambda) \Psi+V(\Psi)] \oplus N(\Psi)=0,
$$

where $T=t \oplus t \oplus \ldots$ ( $A$ times); $t$ is the one-body part of the Hamiltonian, operating in space $h ; \Psi=\psi_{1} \oplus \psi_{2} \oplus \ldots \psi_{A} \in h_{A} ; \Lambda=\lambda_{1} \oplus \lambda_{2} \oplus \ldots \lambda_{A} \in \mathscr{R}_{A}$;

$$
\begin{aligned}
N(\Psi) & =\bigoplus_{k=1}^{A}\left[\left(\psi_{k} \mid \psi_{k}\right)-1\right] ; \\
V(\Psi) & =\bigoplus_{k=1}^{A} \sum_{j=1}^{A}\left(\cdot \otimes \psi_{j} \mid \tilde{v} \psi_{k} \otimes \psi_{j}\right)=\bigoplus_{k} \sum_{j}\left(\cdot \otimes \psi_{k} \mid \hat{v} \psi_{j} \otimes \psi_{j}\right) \\
& =\bigoplus_{k} \sum_{j} \bar{v}\left(\psi_{j}, \psi_{j}, \psi_{k}\right) ;
\end{aligned}
$$

$\tilde{v} \phi \otimes \psi=v(\phi \otimes \psi-\psi \otimes \phi) ; v$ is the two-body part of the Hamiltonian, operating in the space $h \otimes h ; \forall \phi \in h, \forall \omega \in h \otimes h, \psi=(\cdot \otimes \phi \mid \omega) \in h$ is defined by: $(\chi \mid \psi)=(\chi \otimes \phi \mid \omega), \forall \chi \in h ; \Lambda \Psi=\bigoplus_{k=1}^{A} \lambda_{k} \psi_{k}$. The component of Eq. (2.1) in space $h_{A}$ represents the nonlinear eigenvalue equations for the single-particle states $\psi_{k}$ and energies $\lambda_{k}$, the component in space $\mathscr{R}_{A}$ represents the normalization conditions for all $\psi_{k}$. A solution in $\mathscr{H}$ of Eq. (2.1) will be denoted by $\underline{X}=\left\{\underline{\psi}_{k}, \underline{\lambda}_{k}\right\}=\underline{\Psi} \oplus \underline{\Lambda}$.

The operator $t$ is assumed to be self-adjoint and semibounded from below, with lower bound $\tau$. For instance, in the nuclear case, $t$ is just the kinetic energy operator, with $\tau=0$; in the atomic case, it is the kinetic energy plus an attractive Coulomb potential operator, with $\tau=-\frac{1}{2} m c^{2}\left(Z e^{2} / \hbar c\right)^{2}$. In any case, $t-\tau$ is a positive operator. Since the single-particle energies $\underline{\lambda}_{k}$ are negative, the resolvent $\left(t-\tau-\lambda_{k}\right)^{-1}$ is bounded in a neighbourhood of $\underline{\lambda}_{k}$.

In order to ensure the possibility of using the Kantorovic theorem (Theorem 2 of Section 5), the left-hand side of Eq. (2.1) should be twice Fréchet-differentiable in a neighbourhood of $\underline{X}$, so that all linear operators appearing there should be bounded. This can be achieved by transforming Eq. (2.1) by means of the operator $G(\Lambda) \oplus I\left(\mathscr{R}_{A}\right)$, where $G(\Lambda)=\bigoplus_{k=1}^{A}\left(t-\tau-\lambda_{k}\right)^{-1}, \lambda_{k}<0 \forall k, I(\mathscr{X})$ is the identity operator in space $\mathscr{X}$. The transformed equation is

$$
[\Psi+G(\Lambda) U(\Psi)] \oplus N(\Psi)=0,
$$

with $U(\Psi)=\tau \Psi+V(\Psi)$, and will be used in the following. Strictly speaking, on the first $\Psi$ in Eq. (2.2) one should operate with the restriction of the identity operator, defined in the domain $\mathscr{D}(T)$ of $T$, but this restriction can be uniquely extended by continuity to $I\left(h_{A}\right)$. The Eqs. (2.1) and (2.2) are equivalent: it is obvious that any solution of (2.1) is also 
solution of (2.2); it will be shown in Section 4 that the converse is true if $\bar{v}$ is bounded.

The left-hand side of Eq. (2.2) will be denoted by $F(X)$, with $X=\Psi \oplus \Lambda$; it defines a nonlinear operator $F: \mathscr{H} \rightarrow \mathscr{H}$.

\section{Conditions on the Two-body Potential}

The existence of the first and second Fréchet derivatives of $F$ in a neighbourhood of a solution of Eq. (2.2) is expected to depend on the properties of the potential $v$. We are going to examine three conditions on $v$ :

$$
\begin{aligned}
& \bar{v} \in \mathscr{B}\left(h^{3}, h\right), \\
& \tilde{v} \in \mathscr{B}(h \otimes h), \\
& \hat{v} \in \mathscr{B}(h \otimes h) .
\end{aligned}
$$

The first one will be shown in next section to be sufficient for the existence of $F^{\prime}$ and $F^{\prime \prime}$ in a certain region of $\mathscr{H}$. These three conditions are not all independent of each other. We now prove that condition (3.1) follows from (3.2) or (3.3):

$$
\begin{aligned}
|\bar{v}(\phi, \chi, \psi)| & =|(\tilde{v} \psi \otimes \chi) \phi| \leqq|\tilde{v} \psi \otimes \chi||\phi| \leqq\|\tilde{v}\||\psi \otimes \chi||\phi| \\
& =\|\tilde{v}\||\psi||\chi||\phi| \Rightarrow\|\bar{v}\| \leqq\|\tilde{v}\|<\infty .
\end{aligned}
$$

Similarly,

$$
|\bar{v}(\phi, \chi, \psi)|=|(\hat{v} \phi \otimes \chi) \psi| \leqq\|\hat{v}\||\psi||\chi||\psi| \Rightarrow\|\bar{v}\| \leqq\|\hat{v}\|<\infty .
$$

Let us now give the examples of two classes of potentials satisfying some of conditions (3.1), (3.2), and (3.3). Since the conditions are independent of the representation, we need only to verify them in a particular representation. We first consider local potentials defined by a bounded function. Assuming galileian and translational invariance, we have in the coordinate representation

$$
\left(x y|v| x^{\prime} y^{\prime}\right)=\delta\left(x+y-x^{\prime}-y^{\prime}\right) \delta\left(x-y-x^{\prime}+y^{\prime}\right) V(x-y), \quad V \in L^{\infty}\left(\mathscr{R}_{3}\right)
$$

(for simplifying the notation, we have not indicated the spin and isospin quantum numbers, here unessential). All potentials of the class (3.4) satisfy condition (3.2) and hence (3.1), but not (3.3):

$$
\forall \psi \in h \otimes h, \quad|\tilde{v} \psi| \leqq 2|\psi|\|V\|_{\infty} \Rightarrow \tilde{v} \in \mathscr{B}(h \otimes h) .
$$

It is straightforward to verify that (3.3) cannot be satisfied by local potentials. 
Similarly, let us define a class of nonlocal potentials

$$
\begin{gathered}
\left(x y|v| x^{\prime} y^{\prime}\right)=\delta\left(x+y-x^{\prime}-y^{\prime}\right) V\left(x-y, x^{\prime}-y^{\prime}\right), \\
\left|V\left(x, x^{\prime}\right)\right| \leqq A\left(\left|x-x^{\prime}\right|\right) B\left(\left|x+x^{\prime}\right|\right), \\
A, B \in L^{\infty}\left(\mathscr{R}_{3}\right) \cap L^{1}\left(\mathscr{R}_{3}\right) .
\end{gathered}
$$

To prove (3.2) and (3.3) for potentials (3.5), we make use of a sufficient condition for boundedness of operators in spaces $L^{2}$ (see Ref. [11], Section 172):

$$
\begin{aligned}
& \sup _{x, y \in \mathscr{R}_{3}} \int d y^{\prime} d x^{\prime}\left|\left(x y|\tilde{v}| x^{\prime} y^{\prime}\right)\right| \\
& \leqq \min \left\{2\|A\|_{\infty}\|B\|_{1}, 2\|A\|_{1}\|B\|_{\infty},\|A\|_{\infty}\|B\|_{1}+\|A\|_{1}\|B\|_{\infty}\right\}<\infty \\
& \Rightarrow \tilde{v} \in \mathscr{B}(h \otimes h) ; \\
& \sup _{x, x^{\prime} \in \mathscr{R}_{3}} \int d y d y^{\prime}\left|\left(x y|\tilde{v}| x^{\prime} y^{\prime}\right)\right| \leqq\|A\|_{1}\|B\|_{\infty}+\|A\|_{\infty}\|B\|_{1}<\infty \\
& \Rightarrow \hat{v} \in \mathscr{B}(h \otimes h) .
\end{aligned}
$$

We note that for the validity of condition (3.2) either one of the following less stringent requirements is sufficient:

$$
\begin{aligned}
& \left\{A \in L^{\infty}\left(\mathscr{R}_{3}\right), B \in L^{1}\left(\mathscr{R}_{3}\right)\right\}, \\
& \left\{A \in L^{1}\left(\mathscr{R}_{3}\right), B \in L^{\infty}\left(\mathscr{R}_{3}\right)\right\} .
\end{aligned}
$$

We conclude this section by remarking that several nuclear potentials, including the one used in Ref. [6], satisfy condition (3.1). On the other hand, local potentials with $r^{-1}$ singularity at $r=0$ do not satisfy (3.1), so that the present treatment does not hold for them. However, this is not a serious difficulty, because in the case of the Coulomb potential the singularity can be eliminated by considering an extended charge distribution (physically, this is even more realistic), in the nuclear case the potential is nonlocal near $r=0$, where it should rather have the property (3.5). Furthermore, we note that the choice of the Hilbert space $\mathscr{H}$ as the functional space where the solution of the Hartree-Fock equations is sought, is merely a matter of convenience. The main results of our work rest on the Kantorovic theorem, which holds for any Banach space. This suggests the possibility of choosing another functional space, in such a way that potentials with $r^{-1}$ singularity at the origin are bounded operators ${ }^{3}$. In this case, the components $\psi_{k}$ of a solution of Eq. (2.2) would still belong to the Hilbert space $h$, as the physics of the problem requires, because the equation $N(\underline{\Psi})=0$ would still hold.

\footnotetext{
${ }^{3}$ We thank Prof. A. Agodi for this remark.
} 


\section{Fréchet Derivatives}

We are concerned in this section with the existence of the first and second Fréchet derivatives of the nonlinear operator $F$, defined in Section 2. Supposing that $G, U$, and $N$ are twice Fréchet-differentiable in the point $X_{0}=\Psi_{0} \oplus \Lambda_{0}$, we have (by definition of derivative)

$$
F^{\prime}\left(X_{0}\right) X=\left[\Psi+G\left(\Lambda_{0}\right) U^{\prime}\left(\Psi_{0}\right) \Psi+G^{\prime}\left(\Lambda_{0}\right) \Lambda U\left(\Psi_{0}\right)\right] \oplus N^{\prime}\left(\Psi_{0}\right) \Psi,
$$

where $U^{\prime}\left(\Psi_{0}\right) \in \mathscr{B}\left(h_{A}\right), G^{\prime}\left(\Lambda_{0}\right) \in \mathscr{B}\left[\mathscr{R}_{A}, \mathscr{B}\left(h_{A}\right)\right], N^{\prime}\left(\Psi_{0}\right) \in \mathscr{B}\left(h_{A}, \mathscr{R}_{A}\right)$,

$$
F^{\prime}\left(X_{0}\right) \in \mathscr{B}(\mathscr{H})
$$

$$
\begin{aligned}
& F^{\prime \prime}\left(X_{0}\right)(X, Y)=\left[G^{\prime \prime}\left(\Lambda_{0}\right)(\Lambda, \Xi) U\left(\Psi_{0}\right)+G\left(\Lambda_{0}\right) U^{\prime \prime}\left(\Psi_{0}\right)(\Psi, \Phi)\right. \\
& \left.+G^{\prime}\left(\Lambda_{0}\right) \Lambda U^{\prime}\left(\Psi_{0}\right) \Phi+G^{\prime}\left(\Lambda_{0}\right) \Xi U^{\prime}\left(\Psi_{0}\right) \Psi\right] \oplus N^{\prime \prime}\left(\Psi_{0}\right)(\Psi, \Phi),
\end{aligned}
$$

where $U^{\prime \prime}\left(\Psi_{0}\right) \in \mathscr{B}\left(h_{A}{ }^{2}, h_{A}\right), G^{\prime \prime}\left(\Lambda_{0}\right) \in \mathscr{B}\left[\mathscr{R}_{A}{ }^{2}, \mathscr{B}\left(h_{A}\right)\right], N^{\prime \prime}\left(\Psi_{0}\right) \in \mathscr{B}\left(h_{A}{ }^{2}, \mathscr{R}_{A}\right)$, $F^{\prime \prime}\left(X_{0}\right) \in \mathscr{B}\left(\mathscr{H}^{2}, \mathscr{H}\right)$.

We prove the existence of $U^{\prime}, U^{\prime \prime}, G^{\prime}, G^{\prime \prime}, N^{\prime}, N^{\prime \prime}$.

Lemma 1. If condition (3.1) holds, then both $U^{\prime}\left(\Psi_{0}\right)$ and $U^{\prime \prime}\left(\Psi_{0}\right)$ exist for all $\Psi_{0} \in h_{A}$.

Proof. We have for all $\Psi_{0}, \Psi \in h_{A}$ :

$$
\begin{aligned}
\lim _{|\Psi| \rightarrow 0} \mid U\left(\Psi_{0}+\Psi\right)-U\left(\Psi_{0}\right)-\tau \Psi \\
\quad-\left.\bigoplus_{k=1}^{A} \sum_{j=1}^{A}\left[\bar{v}\left(\psi_{j}^{0}, \psi_{j}^{0}, \psi_{k}\right)+\bar{v}\left(\psi_{j}^{0}, \psi_{j}, \psi_{k}^{0}\right)+\bar{v}\left(\psi_{j}, \psi_{j}^{0}, \psi_{k}^{0}\right)\right]|\times| \Psi\right|^{-1}=0 ;
\end{aligned}
$$

by definition, the term $\tau \Psi+\oplus \Sigma[\ldots]$ should therefore be identified with the first differential

$$
U^{\prime}\left(\Psi_{0}\right) \Psi=\tau \Psi+\bigoplus_{k=1}^{A} \sum_{j=1}^{A}\left[\bar{v}\left(\psi_{j}^{0}, \psi_{j}^{0}, \psi_{k}\right)+\bar{v}\left(\psi_{j}^{0}, \psi_{j}, \psi_{k}^{0}\right)+\bar{v}\left(\psi_{j}, \psi_{j}^{0}, \psi_{k}^{0}\right)\right]
$$

The boundedness of the operator $U^{\prime}\left(\Psi_{0}\right)$ follows:

$$
\left\|U^{\prime}\left(\Psi_{0}\right)\right\|=\sup _{\Psi \in h_{A}} \frac{\left|U^{\prime}\left(\Psi_{0}\right) \Psi\right|}{|\Psi|} \leqq|\tau|+3\|\bar{v}\|\left|\Psi_{0}\right|^{2}<\infty \forall \Psi_{0} \in h_{A},
$$

so that $U^{\prime}\left(\Psi_{0}\right)$ is the first Fréchet derivative. Furthermore we have $\forall \Psi_{0}, \Psi, \Phi \in h_{A}$ :

$$
\begin{aligned}
& \lim _{|\Psi| \rightarrow 0,|\Phi| \rightarrow 0} \mid U^{\prime}\left(\Psi_{0}+\Psi\right) \Phi-U^{\prime}\left(\Psi_{0}\right) \Phi-\bigoplus_{k=1}^{A} \sum_{j=1}^{A}\left[\bar{v}\left(\psi_{j}^{0}, \phi_{j}, \psi_{k}\right)\right. \\
& \quad+\bar{v}\left(\psi_{j}^{0}, \psi_{j}, \phi_{k}\right)+\bar{v}\left(\phi_{j}, \psi_{j}^{0}, \psi_{k}\right)+\bar{v}\left(\psi_{j}, \psi_{j}^{0}, \phi_{k}\right)+\bar{v}\left(\phi_{j}, \psi_{j}, \psi_{k}^{0}\right) \\
& \left.\quad+\bar{v}\left(\psi_{j}, \phi_{j}, \psi_{k}^{0}\right)\right]\left.|| \Psi\right|^{-1}|\Phi|^{-1}=0 .
\end{aligned}
$$


The second differential $U^{\prime \prime}\left(\Psi_{0}\right)(\Psi, \Phi)$ is given by the term $\oplus \Sigma[\ldots]$ and the norm of the second derivative $U^{\prime \prime}\left(\Psi_{0}\right)$ is estimated by

$$
\left\|U^{\prime \prime}\left(\Psi_{0}\right)\right\|=\sup _{\Phi, \Psi \in h_{A}} \frac{\left|U^{\prime \prime}\left(\Psi_{0}\right)(\Psi, \Phi)\right|}{|\Psi||\Phi|} \leqq 6\|\bar{v}\|\left|\Psi_{0}\right|<\infty \forall \Psi_{0} \in h_{A} .
$$

This completes the proof of Lemma 1.

Lemma 2. $G^{\prime}(\Lambda)$ and $G^{\prime \prime}(\Lambda)$ exist for all those $\Lambda \in \mathscr{R}_{A}$, such that $\lambda_{k}<0 \forall k$.

Proof. Following the same procedure as in Lemma 1, we find

$$
\begin{aligned}
G^{\prime}\left(\Lambda_{0}\right) \Lambda & =\bigoplus_{k=1}^{A}\left[g\left(\lambda_{k}^{0}\right)\right]^{2} \lambda_{k}, \\
\left\|G^{\prime}\left(\Lambda_{0}\right)\right\| & =\sup _{\Lambda \in \mathscr{R}_{A}} \frac{\left\|G^{\prime}\left(\Lambda_{0}\right) \Lambda\right\|}{|\Lambda|} \leqq \max _{k}\left\|g\left(\lambda_{k}^{0}\right)\right\| \leqq\left(\min _{k}\left|\lambda_{k}^{0}\right|\right)^{-1} \\
<\infty & \forall \lambda_{k}^{0}<0, \\
G^{\prime \prime}\left(\Lambda_{0}\right)(\Lambda, \Xi) & =2 \bigoplus_{k=1}^{A}\left[g\left(\lambda_{k}^{0}\right)\right]^{3} \lambda_{k} \xi_{k}, \\
\left\|G^{\prime \prime}\left(\Lambda_{0}\right)\right\| & =\sup _{\Lambda, \Xi \in \mathscr{R}_{A}} \frac{\left\|G^{\prime \prime}\left(\Lambda_{0}\right)(\Lambda, \Xi)\right\|}{|\Lambda||\Xi|} \leqq 2 \max _{k}\left\|g\left(\lambda_{k}^{0}\right)^{3}\right\| \\
\leqq & 2\left(\min _{k}\left|\lambda_{k}^{0}\right|^{3}\right)^{-1}<\infty \forall \lambda_{k}^{0}<0 .
\end{aligned}
$$

Lemma 3. $N^{\prime}\left(\Psi_{0}\right)$ and $N^{\prime \prime}\left(\Psi_{0}\right)$ exist for all $\Psi_{0} \in h_{A}$.

Proof. As previously, we find

$$
\begin{aligned}
& N^{\prime}\left(\Psi_{0}\right) \Psi=2 \bigoplus_{k=1}^{A}\left(\psi_{k}^{0} \mid \psi_{k}\right), \\
& \left\|N^{\prime}\left(\Psi_{0}\right)\right\|=\sup _{\Psi \in h_{A}} \frac{\left|N^{\prime}\left(\Psi_{0}\right) \Psi\right|}{|\Psi|} \leqq \max _{k}\left|\psi_{k}^{0}\right| \leqq 2\left|\Psi_{0}\right|<\infty \quad \forall \Psi_{0} \in h_{A} ; \\
& N^{\prime \prime}\left(\Psi_{0}\right)(\Phi, \Psi)=2 \bigoplus_{k=1}^{A}\left(\phi_{k} \mid \psi_{k}\right), \\
& \left\|N^{\prime \prime}\left(\Psi_{0}\right)\right\|=\sup _{\Phi, \Psi \in \hbar_{A}} \frac{\left|N^{\prime \prime}\left(\Psi_{0}\right)(\Phi, \Psi)\right|}{|\Phi||\Psi|} \leqq 2 \forall \Psi_{0} \in h_{A} .
\end{aligned}
$$

We are now ready to state and prove a theorem, which is essential for our purpose.

Theorem 1. If condition (3.1) holds, then the operator $F$ defined in Section 2 is twice Fréchet-differentiable, according to Eqs. (4.1)-(4.8), in a 
ball $\Omega_{0}:=\left\{X:\left|X-X_{0}\right| \leqq R\right\}$, with

$$
X_{0}=\left\{\psi_{k}^{0}, \lambda_{k}^{0}\right\}, \lambda_{k}^{0}<0 \forall k ; \quad R=\min _{k}\left|\lambda_{k}^{0}\right|-\varepsilon,\left(\forall \varepsilon: 0<\varepsilon<\min _{k}\left|\lambda_{k}^{0}\right|\right) .
$$

Proof. Remembering Eqs. (4.1) and (4.2), Lemma 1, Lemma 2 and Lemma 3 , we have only to prove the assertion concerning $R$. Putting $\min _{k}\left|\lambda_{k}^{0}\right|=\left|\lambda_{m}^{0}\right|$, we can exclude the case $R=\left|\lambda_{m}^{0}\right|$, because although the vector $\bar{X}=\Psi_{0} \oplus \lambda_{1}^{0} \oplus \ldots \lambda_{m-1}^{0} \oplus 0 \oplus \lambda_{m+1}^{0} \oplus \ldots \lambda_{A}^{0}$ is contained in the closed ball with centre $X_{0}$ and radius $\left|\lambda_{m}^{0}\right|$, the operator $F$ is not Fréchetdifferentiable at $\bar{X}$ (see Lemma 2). A fortiori, we can exclude the case $R>\left|\lambda_{m}^{0}\right|$. In the remaining case $R<\left|\lambda_{m}^{0}\right|$, it is not possible to choose a vector $X$, such that $X \in \Omega_{0}$ and $\lambda_{k} \geqq 0$ for some $k$ (say $k=k_{1}, k_{2} \ldots k_{\mathrm{s}}$ ). Assuming the contrary to be true, it results

$$
\left|\lambda_{m}^{0}\right|-\varepsilon \geqq\left|X-X_{0}\right| \geqq\left|\lambda_{p}^{0}\right|,\left(p=k_{1}, k_{2}, \ldots k_{s}\right),
$$

which can be satisfied only if $\varepsilon=0$. The contradiction proves the assertion.

Now, we can complete the proof of the equivalence of Eq. (2.1) and Eq. (2.2). For a solution $\underline{X}=\underline{\Psi} \oplus \underline{\Lambda}$ of Eq. (2.2) there are two alternatives: either $\underline{\Psi} \in \mathscr{D}(T)$, or $\underline{\Psi} \notin \mathscr{D}(T)$. In the first case, it is evident that $\underline{X}$ is also a solution of Eq. (2.1); the second case can be excluded, because $U$ is continuous (being differentiable), all $\underline{\lambda}_{k}$ are regular values of $t-\tau$, and the range of the resolvent of an operator coincides with the domain of the operator itself (see Ref. [11], Section 189).

\section{Construction of a Solution}

A constructive method for proving the existence and the local uniqueness of a solution of the Hartree-Fock equations is provided by the Newton iteration algorithm, according to the following theorem due to Kantorovič (see Ref. [8], Chapter 18, Section 1, Theorem 6).

Theorem 2. Let $F$ be an operator, mapping the Banach space $\mathscr{X}$ into the Banach space $\mathscr{Y}$, with the following properties:

a) $F$ is twice Fréchet-differentiable for all $X$ in the ball $\Omega_{0}=\left\{X:\left|X-X_{0}\right| \leqq R\right\}$;

b) $F^{\prime}\left(X_{0}\right)$ has a bounded inverse.

Then, putting $h=\eta K$, where $\eta \geqq\left|\left[F^{\prime}\left(X_{0}\right)\right]^{-1} F\left(X_{0}\right)\right|$ and $K \geqq \sup _{X \in \Omega_{0}}$ $\left\|\left[F^{\prime}\left(X_{0}\right)\right]^{-1} F^{\prime \prime}(X)\right\|$, both the Newton sequence

$$
X_{n+1}=X_{n}-\left[F^{\prime}\left(X_{n}\right)\right]^{-1} F\left(X_{n}\right), \quad(n=0,1,2, \ldots)
$$

and the modified Newton sequence

$$
\hat{X}_{n+1}=\hat{X}_{n}-\left[F^{\prime}\left(X_{0}\right)\right]^{-1} F\left(\hat{X}_{n}\right), \quad(n=0,1,2 \ldots)
$$


converge to a solution $\underline{X}$ of the equation $F(X)=0$, if

$$
h \leqq \frac{1}{2}, \quad R \geqq(1-\sqrt{1-2 h}) \eta / h .
$$

The solution $\underline{X}$ is unique in $\Omega_{0}$ if

$$
R \leqq(1+\sqrt{1-2 h}) \eta / h, \quad\left(<\text { in the case } h<\frac{1}{2}\right) .
$$

For the sequence (5.1) the error of approximation is estimated by

$$
\left|\underline{X}-X_{n}\right| \leqq 2^{-n}(2 h)^{2^{n}} \eta / h, \quad(n=0,1,2, \ldots),
$$

for the sequence (5.2) by

$$
\left|\underline{X}-\hat{X}_{n}\right| \leqq(1-\sqrt{1-2 h})^{n+1} \eta / h, \quad(n=0,1,2, \ldots) .
$$

For the application of this theorem to the Hartree-Fock equations one has to prove that an approximation $X_{0}$ to an expected solution can be found, with the properties a), b), (5.3) and (5.4). As already anticipated, this requires in any case some numerical calculations. After choosing $X_{0}$ (a possible criterion is suggested in Ref. [6]), Theorem 1 guarantees that an $R$ can always be found, in such a way that condition a) of Theorem 2 holds. A possible way of checking condition b) is to use the methods for the inverse operator of Refs. [12,13], which can provide also an estimate of the error due to the choice of a subspace of $\mathscr{H}$. Putting $B_{0} K_{0} \geqq K$, with

$$
B_{0} \geqq\left\|\left[F^{\prime}\left(X_{0}\right)\right]^{-1}\right\|, \quad K_{0} \geqq \sup _{X \in \Omega_{0}}\left\|F^{\prime \prime}(X)\right\|,
$$

we can estimate $\eta, K$ and $h . B_{0}$ has to be computed numerically; for $K_{0}$ we can put

$$
\begin{gathered}
K_{0}=2 \max \left\{\left[1+36 \varepsilon^{-2}\|\bar{v}\|^{2}\left(R+\left|\Psi_{0}\right|\right)^{2}\right]^{\frac{1}{2}}, \quad 2 \varepsilon^{-3}\|\bar{v}\|\left(R+\left|\Psi_{0}\right|\right)^{3},\right. \\
\left.3 \varepsilon^{-2}\|\bar{v}\|\left(R+\left|\Psi_{0}\right|\right)^{2}\right\},
\end{gathered}
$$

where $\Omega_{0}, \varepsilon$, and $R$ are defined as in Theorem 1 .

The sequences (5.1) and (5.2) derive from the solution of the equation, representing the best linear approximation to Eq. (2.2) near $X_{0}$ :

$$
F\left(X_{0}\right)+F^{\prime}\left(X_{0}\right)\left(X-X_{0}\right)=0,
$$

or more explicitly

$$
\begin{gathered}
\left\{\Psi+G\left(\Lambda_{0}\right)\left[U\left(\Psi_{0}\right)+U^{\prime}\left(\Psi_{0}\right)\left(\Psi-\Psi_{0}\right)\right]+G^{\prime}\left(\Lambda_{0}\right)\left(\Lambda-\Lambda_{0}\right) U\left(\Psi_{0}\right)\right\} \\
\oplus\left[N\left(\Psi_{0}\right)+N^{\prime}\left(\Psi_{0}\right)\left(\Psi-\Psi_{0}\right)\right]=0 .
\end{gathered}
$$

Equation (5.5) is a Fredholm linear equation of the second kind. If both conditions (3.2) and (3.3) are satisfied, as for potentials (3.5), its kernel belongs to the Hilbert-Schmidt class. 
Transforming Eq. (5.5) by the operator $T-\tau-\Lambda_{0}$ we get the equivalent equation

$$
\begin{gathered}
{\left[\left(T-\tau-\Lambda_{0}\right) \Psi+U\left(\Psi_{0}\right)+U^{\prime}\left(\Psi_{0}\right)\left(\Psi-\Psi_{0}\right)+\left(\Lambda-\Lambda_{0}\right) G\left(\Lambda_{0}\right) U\left(\Psi_{0}\right)\right]} \\
\oplus\left[N\left(\Psi_{0}\right)+N^{\prime}\left(\Psi_{0}\right)\left(\Psi-\Psi_{0}\right)\right]=0,
\end{gathered}
$$

which is (in coordinate representation) a system of integro-differential equations. Still another form of linear approximation to Eq. (2.1) or (2.2) can be used, besides (5.5) and (5.6): by treating formally the left-hand side of Eq. (2.1) as if it were Fréchet-differentiable, we get the linear equation

$$
\begin{aligned}
& {\left[\left(T-\Lambda_{0}\right) \Psi+V\left(\Psi_{0}\right)+V^{\prime}\left(\Psi_{0}\right)\left(\Psi-\Psi_{0}\right)-\left(\Lambda-\Lambda_{0}\right) \Psi_{0}\right] } \\
& \oplus\left[N\left(\Psi_{0}\right)+N^{\prime}\left(\Psi_{0}\right)\left(\Psi-\Psi_{0}\right)\right]=0
\end{aligned}
$$

Subtracting Eq. (5.7) from (5.6), we are left with

$$
\left(\Lambda-\Lambda_{0}\right)\left[\Psi_{0}+G\left(\Lambda_{0}\right) U\left(\Psi_{0}\right)\right]=0,
$$

so that the two equations are equivalent if $X_{0}=\Psi_{0} \oplus \Lambda_{0}$ is already a solution of Eq. (2.2). Nevertheless, if $X_{0}$ is near enough to a solution $\underline{X}$ of Eq. (2.2), then Eqs. (5.6) and (5.7) differ only slightly in the term depending linearly on $X-X_{0}$ and the Newton sequences derived from the two equations converge to the same $\underline{X}$ (see Ref. [8] Chapter 18, Section 2, Theorem 1). This remark can provide a rigorous argument for extending to an infinite-dimensional space the method of Ref. [6], where the numerical calculations for the nucleus ${ }^{16} \mathrm{O}$ have been performed by iterating Eq. (5.7).

We thank Prof. A. Agodi for several discussions and for the critical reading of the manuscript.

\section{Appendix}

We report below in alphabetic order the main symbols and their definition.

$A$ : number of identical fermions.

$\mathscr{B}\left(\mathscr{X}^{n}, \mathscr{Y}\right)$ : Banach space of bounded $n$-linear operators (uniform norm), mapping $\mathscr{X} \times \mathscr{X} \times \cdots \mathscr{X}$ ( $n$ times) into $\mathscr{Y} ;(n=1,2, \ldots)$.

$\mathscr{B}(\mathscr{X})=\mathscr{B}(\mathscr{X}, \mathscr{X})$.

$\mathscr{B}_{2}(h)$ : space of Hilbert-Schmidt operators in $h$.

$F(X)=[\Psi+G(\Lambda) U(\Psi)] \oplus N(\Psi) ; F: \mathscr{H} \rightarrow \mathscr{H}$.

$F^{\prime}(X), F^{\prime \prime}(X)$ : Fréchet derivatives of $F$ at $X$; a similar notation is used for the derivatives of $G, N$, and $U$. 


$$
\begin{aligned}
& g(\lambda)=(t-\tau-\lambda)^{-1} ; g: \mathscr{R} \rightarrow \mathscr{B}(h) . \\
& G(\Lambda)=\bigoplus_{k=1}^{A} g\left(\lambda_{k}\right) ; G: \mathscr{R}_{A} \rightarrow \mathscr{B}\left(h_{A}\right) .
\end{aligned}
$$

$h$ : real Hilbert space of single-particle states.

$h_{A}=h \oplus h \oplus \ldots h(A$ times $)$.

$\mathscr{H}=h_{A} \oplus \mathscr{R}_{A}$.

$I(\mathscr{X})$ : identity operator in $\mathscr{X}$.

$\mathscr{L}(\mathscr{X})$ : vector space of linear operators, mapping $\mathscr{X}$ into itself.

$L^{p}\left(\mathscr{R}_{3}\right)$ : Banach space of functions with integrable $p^{\text {th }}$ power $[8,11]$; $1 \leqq p \leqq \infty$.

$$
N(\Psi)=\bigoplus_{k=1}^{A}\left[\left(\psi_{k} \mid \psi_{k}\right)-1\right] ; N: h_{A} \rightarrow \mathscr{R}_{A}
$$

$\mathscr{R}$ : real numbers.

$\mathscr{R}_{A}=\mathscr{R} \oplus \mathscr{R} \oplus \cdots \mathscr{R}(A$ times $)$.

$t$ : one-body part of Hamiltonian operator in space $h ; t \in \mathscr{L}(h)$.

$T=t \oplus t \oplus \cdots(A$ times $) ; T \in \mathscr{L}\left(h_{A}\right)$.

$U(\Psi)=\tau \Psi+V(\Psi) ; U: h_{A} \rightarrow h_{A}$.

$V(\Psi)=\bigoplus_{k=1}^{A} \sum_{j=1}^{A} \bar{v}\left(\psi_{j}, \psi_{j}, \psi_{k}\right) ; V: h_{A} \rightarrow h_{A}$.

$v$ : two-body part of Hamiltonian operator in space $h \otimes h ; v \in \mathscr{B}(h \otimes h)$.

$\tilde{v}$ : antisymmetrized $v: \tilde{v} \phi \otimes \psi=v(\phi \otimes \psi-\psi \otimes \phi) \in h \otimes h \equiv \mathscr{B}_{2}(h)$; $\tilde{v} \in \mathscr{B}(h \otimes h)$.

$\hat{v}:(\phi \otimes \chi \mid \hat{v} \psi \otimes \omega)=(\phi \otimes \psi \mid \tilde{v} \chi \otimes \omega)$.

$\bar{v}: \bar{v}(\phi, \chi, \psi)=(\hat{v} \phi \otimes \chi) \psi=(\tilde{v} \psi \otimes \chi) \phi=(\cdot \otimes \psi \mid \hat{v} \phi \otimes \chi)=(\cdot \otimes \phi \mid \tilde{v} \psi \otimes \chi) ;$ $\bar{v} \in \mathscr{B}\left(h^{3}, h\right)$.

$X=\Psi \oplus \Lambda \in \mathscr{H} ; Y=\Phi \oplus \Xi \in \mathscr{H}$.

$\mathscr{X}, \mathscr{Y}$ : unspecified Banach spaces.

$\lambda, \xi \in \mathscr{R}$.

$\Lambda, \Xi \in \mathscr{R}_{A} ; \Lambda=\lambda_{1} \oplus \lambda_{2} \oplus \cdots \lambda_{A} ; \Xi=\xi_{1} \oplus \xi_{2} \oplus \cdots \xi_{A}$.

$\Lambda \Psi=\bigoplus_{k=1}^{A} \lambda_{k} \psi_{k}$

$\tau$ : finite lower bound of $t$.

$\phi, \chi, \psi, \omega \in h$.

$\Phi=\phi_{1} \oplus \phi_{2} \oplus \cdots \phi_{A} \in h_{A} ; \Psi=\psi_{1} \oplus \psi_{2} \oplus \cdots \psi_{A} \in h_{A}$.

$|\cdot|$ : norm of vectors in Banach space $\mathscr{X}$. In Hilbert spaces: scalar product norm.

$\|\cdot\|:$ uniform norm of operators of $\mathscr{B}\left(\mathscr{X}^{n}, \mathscr{Y}\right),(n=1,2,3)$.

$(\cdot 1 \cdot)$ : scalar product in space $h$ or $h \otimes h$.

$\langle X \mid Y\rangle=\sum_{k=1}^{A}\left[\left(\psi_{k} \mid \phi_{k}\right)+\lambda_{k} \xi_{k}\right]:$ scalar product in $\mathscr{H}$.

$\|\cdot\|_{p}:$ norm in space $L^{p}\left(\mathscr{R}_{3}\right)$. 


\title{
References
}

1. Hartree, D. R.: Proc. Cambr. Phil. Soc. 24, 89 (1927-28); V. Fock, Z. Phys. 61, 126 (1930)

2. Hartree, D. R.: The calculation of atomic structures, New York: J. Wiley 1957

3. Kerman,A.K.: Proc. of International Conference on properties of nuclear states, ed. by M. Harvey, Presses de l'Université de Montréal, 1969

4. Moshinsky, M.: Amer. Journ. Phys. 36, 52-53 (1968); The harmonic oscillator in modern physics: from atoms to quarks. New York: Gordon \& Breach 1969

5. Reeken, M.: Journ. Math. Phys. 11, 2505-2512 (1970)

6. Catara, F., Di Toro, M., Pace, E., Schiffrer, G.: Nuovo Cim. 11 A, $733-748$ (1972)

7. Kantorovič, L.V.: Usp. Mat. Nauk 3, 89-185 (1948)

8. Kantorovič, L.V., Akilov, G.P.: Funkcional'nyj analiz $\mathrm{v}$ normirovannyh prostranstvah, Moskva: Fizmatgiz 1959; English transl.: Functional analysis in normed spaces, Oxford: Pergamon Press 1964

9. Schiffrer, G.: Proc. of Symposium on present status and novel developments in the nuclear many-body problem, (Rome, 19-23 sept. 1972), ed. by F. Calogero and C. Ciofi degli Atti, to be published.

10. Catara,F., Di Toro, M., Lombardo, U.: Lett. Nuovo Cim. 4, 849-852 (1972)

11. Smirnov, V.I.: Kurs vysšej matematiki, tom 5, Moskva: Fizmatgiz 1960; English transl.: A course of higher mathematics, vol. 5, Oxford: Pergamon Press 1964

12. Lučka, A. Ju.: Teorija i primenenie metoda osrednenija funkcional'nyh popravok, Kiev: AN USSR 1963; English transl.: The method of averaging functional corrections, New York: Academic Press 1965

13. Rall,L.B.: Computational solution of nonlinear operator equations, New York: J. Wiley 1969

\author{
G. Fonte \\ Istituto di Fisica Teorica \\ dell'Università \\ 57, Corso Italia \\ I-95129 Catania, Italia
}

